\title{
Diacronie
}

Studi di Storia Contemporanea

$N^{\circ} 7,3 \mid 2011$

«Spagna Anno Zero»: la guerra come soluzione

\section{La guerre d'Espagne (sur)exposée dans la presse française}

Étude locale de la presse du département de la Loire (42)

\section{Pascale Moiron}

\section{(2) OpenEdition}

\section{Journals}

Édition électronique

URL : http://journals.openedition.org/diacronie/3447

DOI : 10.4000/diacronie.3447

ISSN : 2038-0925

Éditeur

Association culturelle Diacronie

Référence électronique

Pascale Moiron, "La guerre d'Espagne (sur)exposée dans la presse française », Diacronie [En ligne], N 7,3 | 2011, document 11, mis en ligne le 29 juillet 2011, consulté le 19 avril 2019. URL : http:// journals.openedition.org/diacronie/3447 ; DOI : 10.4000/diacronie.3447 


\section{Diacronie}

N. 7 | 7|2011 Spagna Anno Zero: la guerra come soluzione

11/

\section{La guerre d'Espagne (sur)exposée dans la presse française. Etude locale de la presse du département de la Loire (42)}

Pascale MOIRON*

L'objectif de l'article sera de démontrer comment les habitants de la Loire, pourtant loin des Pyrénées, ont pu suivre tous les événements de la Guerre d'Espagne et se sentir concernéa à travera lea journaux de leur département. En fonction de la tendance politique du journal (communiate, radical, aocio-démocrate, conaervateur), lea analyses produites alimentent la peur de l'encerclement par des dictatures, la peur du bolchéviame qui attaque lea lieux de cultea, la peur, enfin, provoquée par lea aouffrancea dea civila, victimea dea bombardementa Cette peur induit dea réactiona et dea comportementa différenta: dea collectea ainai que dea appela à dea dona aont organiaéa, dea convoia partent de Saint-Etienne vers l'Espagne, des enfants basques sont accueillis en 1937... D'autres utilisent les tensions entre les républicains eapagnola pour dénigrer la gauche françaiae. 


\section{Présentation de la presse ligérienne}

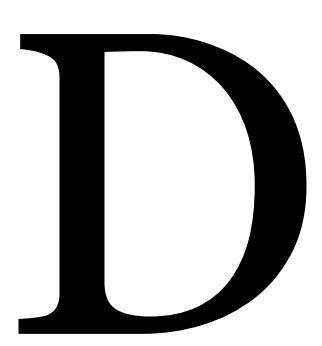

ans les années 30, la presse est le média le plus influent sur l'opinion publique. Elle est la principale source d'informations dans les foyers, même si elle est progressivement concurrencée par la radio. Néanmoins, la lecture du journal reste un geste du quotidien. Et ce journal est le plus souvent issu de la presse locale, la grande presse parisienne ( «e Figaro », « Le Temps », « La République », «L’Humanité ») étant moins influente en province. Dans la Loire, les journaux quotidiens les plus lus sont « La Tribune » et «La Loire Républicaine ». Ces journaux fondés à la fin du XIX̀̀me siècle, «l'âge d'or de la presse française (18711914) $»^{2}$, sont de tendance socio-démocrate pour «La Tribune », radicale pour «La Loire Républicaine ». « La Tribune » a le tirage le plus important (250.00o exemplaires en 19363) et est distribué dans 23 départements. Ce journal est à place égale avec d'autres journaux régionaux comme " Le Progrès de Lyon » (220.000 exemplaires en 1939) ou "La dépêche de Toulouse » (270.00o exemplaires) ${ }^{4}$. Il est dirigé par Louis Soulié, maire de St-Etienne en 1936 dont l'équipe municipale appartient au Front populaire. « La Loire Républicaine » (20.0oo exemplaires) est un journal plus local, de tendance politique radicale (centre). Notre étude s'appuiera donc plus sur son analyse. «Le Mémorial » est aussi un héritage de la fin du XIX ${ }^{\text {ème }}$ siècle. C'est un journal de la droite catholique, un "journal de campagne" 5 , lu également en Ardèche et en Bourgogne. Il est financé par la bourgeoisie de St-Etienne, issue de l’industrie du ruban. Dans les années '30, son ton se radicalise et penche vers l'extrême-droite, ses tirages augmentent de 35.000 exemplaires à 43.500 en 1939.

Notre étude reposera également sur le décryptage de deux hebdomadaires : le premier est «L'Avenir de la Loire» dont la parution est le dimanche (19.500 exemplaires). Le rédacteur en chef, Auguste Prénat, est un avocat de St-Etienne, catholique fervent et défenseur de l'école libre. Ce journal, à la différence du «Mémorial », reste modéré, conservateur certes, mais démocrate-chrétien. Le deuxième hebdomadaire, "Le Cri du peuple », de tendance communiste, est créé en mai 1935. Il se présente comme l' « organe hebdomadaire des ouvriers et des paysans de

\footnotetext{
ALBERT, Pierre, Histoire de la presse, 11·édi., Paris, Que sais-je ?, PUF, 1970, 2010.

Ibidem, p. 99.

LUIRARD, Monique, La régions stéphanoise dans la guerre et dans la paix (1936-1951), St Etienne, Centre d'études foréziennes, 1980, p. 107.

4 ALBERT, Pierre, Histoire de la presse, op. cit.,p. 64.

5 LUIRARD, Monique, La régions stéphanoise, op. cit., p. 108.
} 
la région de le Loire, publié par le Parti communiste» ${ }^{6}$. Son jour de parution est le samedi.

\section{La Guerre d'Espagne dans la presse ligérienne}

L'omniprésence de la Guerre d'Espagne dans la presse française est un fait indéniable, et il en est de même pour la presse locale du département de la Loire. Le décompte du nombre de Une, de photographies et du nombre total d'articles par mois, concernant la Guerre d'Espagne dans « La Loire Républicaine », journal ligérien de tendance radicale, permet une analyse quantitative du phénomène. En effet, les événements d'Espagne peuvent être traités à la Une, dans les pages internationales appelées "Dernière Heure", ou encore, dans les pages locales avec des informations concrètes données sur l'organisation d'aide aux républicains qui sera vue ultérieurement, comme l'accueil de réfugiés.

\section{Photographies et articles sur la Guerre d'Espagne dans le journal La Loire Républicaine}

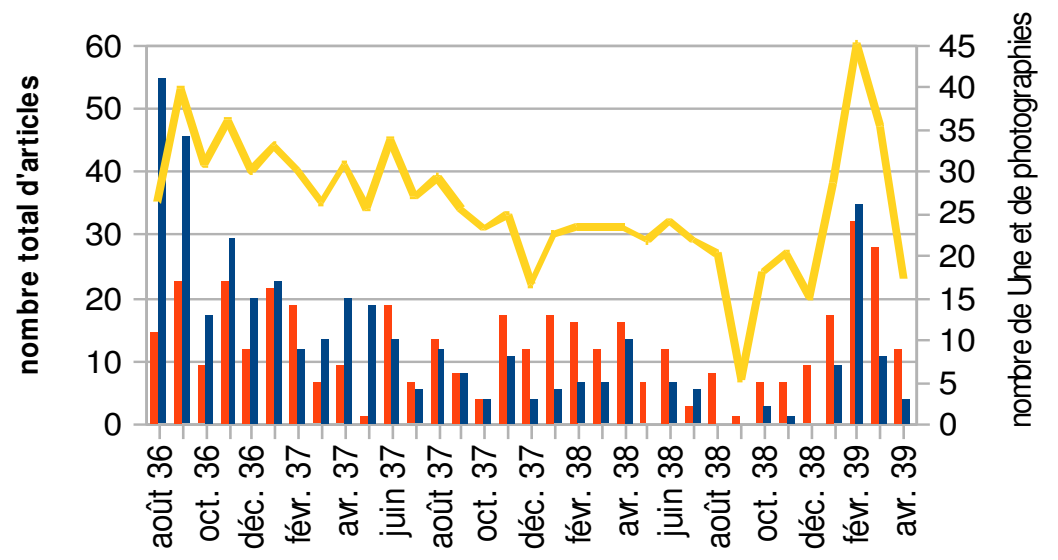

Articles à la Une

Photographies Nombre total d'articles

D'août $1936^{7}$ à août 1937, la moyenne dépasse les 40 articles par mois, mais le nombre est constamment décroissant. En effet, le golpe du 17 au 18 juillet 1936, organisé par les militaires au départ du Maroc surprend et passionne. Tous les fronts de

\footnotetext{
6 Archives départementales de la Loire, PER 28-5 : cette phrase est située en dessous du titre du journal à chaque Une.

$7 \quad$ Le mois de juillet 1936 n'est pas présent car le golpe de Franco a eu lieu le 18 juillet 1936, et le graphique ne prend en compte que des mois complets.
} 
la guerre civile sont alors détaillés : en Andalousie, au pays basque, à Madrid, aux îles Baléares et dans le reste de la Catalogne. Les articles de la "Dernière Heure" mettent en avant l'événement capital : Prise de Badajoz, le 14 août, annoncée le 16 août 1936, Les nationalistes entrent dans Irun, le 2 septembre 1936, dans le journal du 5 septembre, St Sébastien est tombé le 13, annoncé le 15, etc. ; mais ils n’oublient pas de donner des nouvelles des autres fronts également. Quelques erreurs d'information sont à noter : par exemple, celle de Cordoue tombe dans l'article du 25 août marque une faute d'appréciation. La ville andalouse était déjà dans les mains des nationalistes depuis le 18 juillet, et le 20 août, c'est l'échec de la reconquête par les 10.000 miliciens commandés par Miaja ${ }^{8}$. Ces erreurs seront à quantifier dans des recherches ultérieures. Chaque événement important provoque une augmentation du nombre de Une très visible dans le graphique : la bataille de Madrid en novembre 1936, la non-intervention en janvier 1937, le front basque et les tensions à Barcelone en mai et juin 1937.

Enfin, les photographies sont omniprésentes : elles sont au nombre de 41 en août 1936 et de 34 en septembre 1936. Accompagnant la Une, elles représentent les hommes politiques, les lieux de batailles, les hommes armés, miliciens ou nationalistes. Ces photographies contribuent à la construction d'une représentation collective de la guerre civile en Espagne. La communication par l'image s'est réellement développée avec le traitement journalistique de la Guerre d'Espagne. Les préjugés à l'égard de la photographie, la présentant comme un document pour illettré, vont décroissants ${ }^{9}$. Les progrès technologiques (appareils plus maniables comme le Leïca, communication rapide des photographies...) démultiplient les prises. Comme Susan Sontag explique dans un récent article dans le New-Yorker (9 Décembre, 2002), «la guerre civile espagnole a été la première guerre témoin ( « couverte ») dans le sens moderne du terme : par un corps de photographes professionnels au niveau des lignes de l'engagement militaire et dans les villes sous les bombardements, dont le travail a été immédiatement vu dans les journaux et magazines en Espagne et à l'étranger ${ }^{10}$. Dans les Unes des journaux ligériens, elles sont associées à des titres qui ont un effet de choc sur le lecteur. Les deux associés interpellent en frappant les esprits.

De septembre 1937 à décembre 1938, le fléchissement laisse place à une stagnation du nombre total d'articles avec une moyenne qui reste tout de même de 30 articles par mois. Toutefois, la courbe trace deux abîmes, car d'autres événements arrivent à prendre la Une à la Guerre d'Espagne : lors de la guerre sino-japonaise, la prise de

8 HUGH, Thomas, La Guerre d'Espagne, Paris, Robert Laffont, 1961, p.260.

9 FONTAINE, François, Un déluge de feu et d'images, Paris, BDIC/BERG International, 2003.

10 CRAWFORD, Matt, The Spanish Civil War and War Photography in the 1930s, article en ligne tiré de URL: < http://libraries.ucsd.edu/speccoll/swphotojournalism/ > [consulté le 26/07/2011]. 
Nankin en décembre 1937 ; puis, surtout, en septembre 1938, la crise des Sudètes jusqu'à la Conférence de Munich du 29 au 30 septembre. Dès lors, durant l'automne 1938, la presse ligérienne exprime la peur de l'encerclement de la France par des dictatures, à l'Est avec l'Allemagne nazie d'Hitler et l'Italie fasciste de Mussolini, à l'Ouest avec Franco. Cette baisse est très nette pour les photographies : très peu concernent la guerre civile espagnole d'août à décembre 1938. La présentation écrite reste donc plus concernée, plus fidèle ; les photographies affichent une communication plus volatile, n'illustrant que les sujets faisant les gros titres.

Enfin, avec les mois de janvier et février 1939 et les avancées franquistes sur le front de l'Ebre, puis en Catalogne, provoquant la retirada et l'organisation de l'accueil des réfugiés espagnols, la Guerre d'Espagne reprend la Une. Est à noter le volume important des photographies sur l'exil en février 1938 : elles sont au nombre de 26. Toutefois, il ne dépasse pas celui des mois d'août 1936 (41) et de septembre 1936 (34) lors de la mise en place des zones franquistes et des zones républicaines. Dans les pages locales, un sujet conséquent à l'exil est traité avec minutie : les arrivées de réfugiés espagnols accueillis dans La Loire, mais aussi en Haute-Loire. Des reportages photographiques sont même menés localement. Quant à La Une, elle est accaparée par les négociations franco-espagnoles avec toujours cette peur lancinante de l'encerclement de la France par Franco, Mussolini et Hitler. Les accords BérardJordana et le départ de Pétain pour l'ambassade française à Burgos sont accueillis avec soulagement le 27 février et le 5 mars 1939.

\section{Les articles sur le printemps 1937}

Afin de mieux confronter les informations et les analyses des événements espagnols produites par les différents journaux ligériens, cibler une période précise semble nécessaire. En effet, tous ces journaux ont accès aux mêmes informations fournies par l'agence Reuter ou Havas, seule l'interprétation diffère. Dans l'étude de la presse régionale languedocienne, Daniel Meyran conclue: «Ainsi le déchaînement de la guerre civile en Espagne va-t-il entraîner un flot d'informations et de notes d'agences identiques pour les deux journaux ("L'Eclair" et "Le Petit Méridional"), mais les titres et la substance de l'information, c'est-à-dire l'interprétation au niveau du discours, changent selon l'opinion politique de l'un ou de l'autre. Idéologie oblige ${ }^{11}$. La période

11 MEYRAN, Daniel, «La presse régionale languedocienne et les débuts de la Guerre d'Espagne », in Les Français et la Guerre d'Espagne, Actes du colloque de Perpignan, Perpignan, Presses Universitaires de Perpignan, 1990, p. 105. 
sélectionnée, le printemps 1937, est l'une des plus denses dans son traitement journalistique. Les articles de cette période traitent de la chute du pays basque et des troubles à Barcelone entre la CNT (Conderacion Nacional del Trabajo) et le PCE (Partido Comunista de España) ${ }^{12}$. Leur étude permettra d'évaluer la fonction de représentation du discours journalistique. Dans l'analyse du discours de Michel Foucault, il s'agit de la traduction idéologique de l'événement qui lui donne plus de sens vis-à-vis d'un public pas directement concerné. En liant les événements espagnols à l'histoire locale ou nationale, les journalistes décryptent pour leurs lecteurs, au risque de les fausser, les informations données. Ils provoquent également un intérêt supplémentaire car le lecteur se sent alors directement concerné, l'événement n’est plus lointain. Au contraire, il entre dans le quotidien et le prisme de la proximité devient un truchement pour comprendre le monde qui l'entoure.

\subsection{Guernica, avril 1937}

Fig. 1

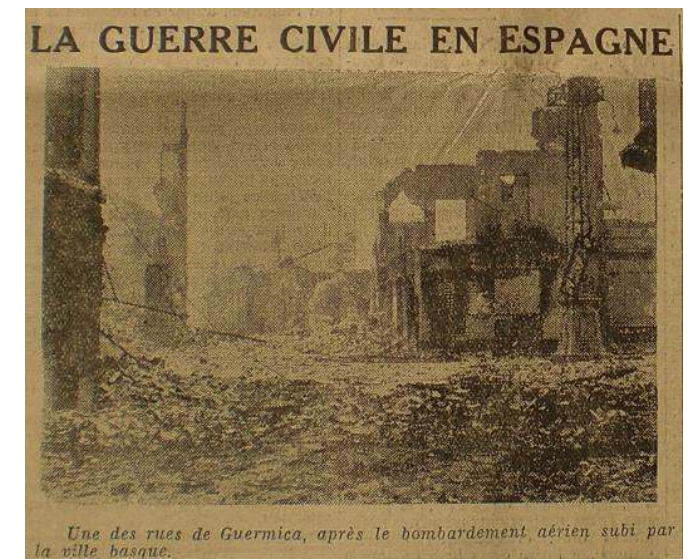

La Une de la Loire Républicaine du $1^{\text {er }}$ mai 1937.

Le bombardement de Guernica, le 26 avril 1937, est l'événement-symbole de la Guerre d'Espagne. Il a fait l'objet de plusieurs photographies qui ont marqué et sont restées présentes dans la mémoire collective ${ }^{13}$. A la Une de « La Loire Républicaine », seule la photographie de la ville détruite est présente sous le titre La Guerre civile en Espagne. L'association entre les deux se fait sans l'aide d'aucun texte, l'identification de

12 CNT (Confédération Nationale du Travail) et PCE (Parti Communiste Espagnol).

13 "[...] courant de pensée continu puisqu'elle ne retient du passé que ce qui en est encore vivant dans la conscience du groupe qu'elle entretient» (HALBWACHS, Maurice, La mémoire collective, Paris, Albin Michel, 1997). 
la Guerre d'Espagne à un paysage urbain dévasté semble aller de soi. L'article Guernica n'existe plus se trouve dans la partie "Dernière heure". Ce désastre génère un sentiment de peur : il rappelle la capacité de "brutalisation"14 de l'homme par l'homme, comme lors de la $1^{\text {ère }}$ Guerre mondiale.

«Le Mémorial » du $1^{\text {er }}$ et 2 mai 1937 possède également, à la Une, une photographie de Guernica, avec la légende "une vue des ruines de Guernica", mais ce n'est pas la même que celle de « La Loire Républicaine ». Le titre de l'article qui l'accompagne est sans lien direct : Le général Franco, acclamé par les troupes et la population, a visité le front de Biscaye. Toutefois, un paragraphe de l'article répond au sujet évoqué par la photographie : «On peut dire sans exagération que Guernica n'existe plus. L’incendie en a fait de décombres innombrables d'où émergent encore quelques pans de murs. »15 Mais, aucune explication n'est donnée sur l'origine de la catastrophe, de ce soit disant incendie. Un article, dans le journal du 7 mai 1937, évoque l'investigation d'un journaliste conservateur, envoyé spécial du « Times », publiée le 5 mai : "A Guernica, on retrouve peu d'éclats de bombes. Les façades des maisons, quand elles sont encore debout, ne portent pas de traces de bombardement». Ces propos sont démentis le lendemain, par la retranscription de l'article du correspondant du « Times » à Bilbao : ce démenti est placé à la Une, mais en fin d'article et de manière assez brève, voire, assez discrète.

L'analyse d'Auguste Prénat dans « L'Avenir de la Loire » est moins impartiale car il cite toutes les informations concernant Guernica, mais le doute persiste : « La TSF officielle et les journaux [...] ont affirmé avec des détails horribles et terrifiants que des avions allemands au service du général Franco, avaient fait pleuvoir des torpilles et des bombes qui avaient fait des femmes et enfants le plus odieux des massacres. Le Gouvernement de Burgos a répondu que Guernica avait été volontairement incendiée au pétrole par les rouges obligés de l'évacuer à la hâte. Qui dit vrai ? » Suit alors, une association avec un événement local : «Guernica est loin, mais Lyon est près. Le petit Gignoux est mort. A-t-il été tué par les pierres que lui ont jetées les écoliers rouges? Est-il mort de peur, parce qu'il était cardiaque ? ${ }^{16}$ Cela permet de maintenir une suspicion envers les "rouges” et alimenter une peur vis-à-vis de la violence déjà présente sur le sol français, même si l'événement local est en rien comparable avec les 1800 morts de Guernica.

«Le Cri du Peuple » parle de Guernica dans le journal du 14 mai 1937, avec donc

14 Concept de George Mosse in De la Grande guerre aux totalitarisme, la brutalisation des sociétés européennes, Paris, Hachette Littératures, Pluriel, 1990.

15 AD Loire, PER 328-91 : Le Mémorial, 1-2 mai 1937.

16 AD Loire PER 9-19 : L'Avenir de la Loire, année 1937, journal du 9 mai. 
une semaine de retard par rapport aux autres journaux ligériens. La responsabilité du massacre ne fait aucun doute: "Il me souvient d'un large titre du "Nouvelliste" affirmant en substance : "Ce sont le rouges qui ont détruit Guernica”. La monstruosité ! Ignore-t-il, ce rédacteur très chrétien du "Nouvelliste" que le peuple basque est profondément catholique? » Le but est clairement de rapprocher les catholiques des communistes: «Là-bas au pays basque, les catholiques, les communistes, les républicains luttent ensemble ». Cette association doit se traduire concrètement avec le Comité de Bilbao auquel les catholiques sont appelés à se joindre, et dont les objectifs politiques seront vus ultérieurement. Ainsi, comme dans "L’Avenir de la Loire », l'événement basque est-il exploité localement. Mais, la peur, cette fois-ci, n'est pas utilisée pour diviser, mais au contraire pour rassembler.

Un mois plus tard, lors de l'accueil de civils basques à St-Etienne, la question de Guernica réapparaît. Le journaliste de «La Loire Républicaine » interviewe une réfugiée « aux cheveux d'or » qui parle Français : " - Guernica a donc été pulvérisée par les torpilles des aviateurs ? - Oui, monsieur ! répond notre interlocutrice. Il y a parmi nous plusieurs habitants de Guernica. Interrogez-les. Leur témoignage est formel. La ville a été détruite en cendres par l'aviation. ${ }^{17}$ Le rôle de l'aviation devient certain, celui des Allemands n'est pas encore évoqué. Une autre réponse est donnée par «Le Mémorial » qui semble interviewer la même personne, blonde, parlant le Français et qui devait sans doute servir d’interprète : «- Et Guernica? - Guernica a été bombardée et brûlée par les avions nationalistes, mais c'était des appareils et pilotes italiens ou allemands ! Diable que voilà une assertion dangereuse pour les relations diplomatiques. $»^{18}$ La peur induite par la pression des dictatures à l'Est de la France reste prégnante.

\subsection{La chute de Bilbao}

L'avancée des franquistes dans le pays basque fait l'objet d'articles à la Une du journal «Le Mémorial ", de manière très régulière: Les nationaux victorieux en Biscaye, le 8 avril 1937, La rupture du Front basque annoncée, le 26 avril, Les nationalistes se sont emparés de la ville et du port de Bermeo, le 3 mai 1937, Les nationalistes dominent Bilbao, le 10 mai, Les nationaux à $3 \mathrm{~km}$ d'El Gallo, dernier boulevard défensif de Bilbao, le 14, Les nationalistes s'emparent des dernières

17 AD Loire, PER 325-73 : La Loire républicain, 10 juin 1937.

18 AD Loire, PER 328-91 : Le Mémorial, 14 juin 1937. 
défenses de Bilbao, le 19, etc. Le suspens est maintenu sur tout le mois de mai, puis s'essouffle. L'annonce réitérée dans 21 articles à la Une d'une victoire attendue des nationalistes semble finir par lasser. Le 14 juin 1937, à nouveau Les nationalistes sont à $3 \mathrm{~km}$ de Bilbao, et le 20 juin est annoncé : Les troupes nationalistes ont conquis Bilbao. L'article est accompagné d'une photographie, encore un paysage urbain en ruines, avec pour légende: «Les ruines de la petite ville d'Amorebieta qui fut incendiée par les milices rouges avant leur retraite ${ }^{19}$. Le but est sans doute de faire le pendant de Guernica, de trouver une ville symbole de la violence rouge et d'en construire une mémoire collective. L'événement avait déjà été traité dans le journal du 20 mai 1937 avec pour titre à la Une: Après avoir dynamité et incendié plusieurs édifices d'Amorebieta, les "rouges" ont tiré à la mitrailleuse sur la population civile. Cela évitait de parler des avancées de l'enquête sur le bombardement de Guernica: le discours journalistique déconstruit l'information pour construire une mémoire partielle et partiale des événements espagnols. Ce procédé est d'autant plus pernicieux qu'il est camouflé par une multitude d’informations détaillées sur les affrontements militaires. Chaque article précise et énumère les localités conquises : « Les troupes des brigades de Navarre se sont emparés des villages d’Aldaye, Frunis, Endecoa, Meacaz, Gorga et Meaca. ${ }^{20}$ Ce qui accrédite les propos grâce à la formulation, celle du discours d'expert. Pourtant, nulle trace de ce village transparaît dans les livres traitant de la Guerre d'Esapgne : Thomas Hugh ne fait aucune allusion à Amorebieta ; et il en est de même pour Bartolomé Bennassar et Antony Beevor ${ }^{21}$. Le village est seulement présent dans une carte sur la campagne de Biscaye (mars-juin 1937), mise en annexe dans l'ouvrage de Bennassar.

Dans «La Loire républicaine » et «Le Cri du Peuple », le front basque est moins surreprésenté dans les Unes. « La Loire républicaine » évoque d’autres sujets comme la question du contrôle de la mer Méditerranée avec, par exemple, le bombardement du port d'Almeria. Mais, les articles de la "Dernière heure" suivent avec la même passion les événements basques avec 25 articles. Dans « Le Cri du Peuple», le traitement du front basque est à l'opposé de celle du «Mémorial»: Sur tous les fronts, les républicains prennent l'offensive, le 15 avril 1937, Le retrait des troupes fascistes d'Espagne battus sur tous les fronts, le 22 avril $^{22}$. Si le sujet devient omniprésent, il prend une forme totalement différente : celle de l'appel aux dons pour le comité de

19 AD Loire, PER 328-91: Le Mémorial, de janvier à juin 1937.

20 AD Loire, PER 328-91 : Le Mémorial, journal du 22 mai 1937.

21 BENNASSAR, Bartolomé, La Guerre d'Espagne et ses lendemains, Paris, Editions Perrin, 2006 et BEEVOR, Antony, La Guerre d'Espagne, Paris, Calmann-Lévy, 2006.

22 AD Loire, PER 28 - 6 : Le Cri du Peuple, 1937. 
Bilbao. Seul l'aspect répétitif du sujet se retrouve avec, jusqu'à la chute de Bilbao annoncée dans le journal du 24 juin, le bilan des dons avec le total des sommes reçues et le nom des donateurs donné à chaque édition.

\subsection{Les événements de Barcelone}

Force est de constater que les événements de Barcelone n'apparaissent à aucun moment dans «Le Cri du Peuple ». Du 2 au 7 mai 1937, des combats opposèrent les libertaires aux communistes. Le gouvernement central envoya des troupes et la CNT lança un appel au calme. Au sein du gouvernement, ces événements provoquèrent une crise, les communistes accusant le POUM (Partido Obrero de la Unificacion marxista) ${ }^{23}$ d'être à l'origine des émeutes. Francisco Largo Caballero (1869-1946) refusant d'ordonner des sanctions, les communistes quittèrent le gouvernement provoquant sa chute, le 15 mai 1937. Juan Negrin Lopez (1887-1956) fut alors chargé de constituer le gouvernement dans lequel ils gagnèrent en influence. Cette image de division, d'exclusion, de prise progressive du pouvoir par les communistes ne coïncide pas avec la politique locale de rassemblement menée par le PCF (Parti communiste français), par le truchement du Comité de Bilbao. Ainsi, est-elle absente dans la presse communiste locale.

A l'inverse, «Le Mémorial » traite immédiatement du sujet : le journal du 8 mai 1937 évoque des émeutes anarchistes en Catalogne avec le titre L'état anarchique s'aggrave à Barcelone et s'étend à d'autres régions de la Généralité de Catalogne. Ces termes insinuent un lien consubstantiel entre anarchistes et situation anarchique, et conséquemment, violente: «De sérieux désordres eurent lieu à nouveau jeudi et vendredi. On calcule qu'il y a déjà près de 400 morts et un milliers de blessés » (journal du 9 mai). La présentation des divisions entre républicains ne fait aucune allusion aux communistes : "Actuellement, toute la question est de savoir si Valence est vraiment décidé à aller jusqu'au bout. Si oui, le gouvernement Largo Caballero devra envoyer à Barcelone une partie de la garde prétorienne qu'il a organisé pendant ces derniers mois. Et il y aura une lutte d'autant plus sanglante que les anarchistes désertent le front d'Aragon pour venir au secours et apporter leur renfort à leur camarade de Barcelone ». Dans le journal du 10 mai, le front basque prend la Une et les événements de Barcelone sont relégués à la partie "Dernière Heure" dans un article intitulé : La Semaine rouge de Barcelone qui reprend les informations dites précédemment mais avec plus de

23 POUM (Parti Ouvrier d'Unification Marxiste), parti jugé trotskiste par Moscou. 
détails. Le changement de gouvernement fait la Une des journaux du 17 et 18 mai 1937. Néanmoins, il faut attendre le 18 mai, pour que le rôle du PCE dans ces tensions soit mentionné, dans un article de Louis Chauvet, issu de la rédaction parisienne : "Encore une preuve de l'ingérence des soviets dans les affaires d'Espagne : c'est l'ambassadeur de l'URSS à Valence qui a provoqué la nouvelle crise ministérielle et la démission de Largo Caballero ! L'action punitive contre les anarchistes et les trotskystes responsables du dernier soulèvement en Catalogne lui avait paru très insuffisante. Il exigea des mesures draconiennes! Les communistes veulent acquérir enfin l’influence prépondérante que les libertaires ont exercée jusqu'à ce jour. Quant à la république, elle tombe de Charybde en Scylla ». Effectivement, l'URSS joua un rôle important dans la répression: Orlov, chef du $\mathrm{NKVD}^{24}$ en Espagne, a mis au point un stratagème afin de démontrer la trahison du POUM et de son chef Andreu Nin (1892-1937). Mais, cet abus de pouvoir fut condamné par José Diaz (1896-1942), l'un des cadres du PCE²5.

Il en est de même pour "La Loire républicaine » : ce sont surtout les violences commises par les anarchistes qui sont mises en avant : Les anarchistes ont mis la ville à feu et à sang, (6 mai 1937), Les anarchistes sèment la terreur, (9 mai), etc. Un témoignage publié par le journal « Le Matin » est cité : « Je viens de quitter Barcelone. La terreur y est complète. Tout est arrêté : tramways, autobus, téléphones, la plupart des boutiques ont fermé leur porte, sauf quelques cafés connus pour être le lieu de rendez-vous des extrémistes. Les habitants sont terrifiés : songez que dans les artères de la ville, 12 autos blindées, appartenant aux extrémistes, aux anarchistes, patrouillent jour et nuit, et ouvrent le feu à tout propos». ${ }^{26}$

Ainsi, l'analyse des journaux ligériens permet de conclure sur la divergence de lecture des événements de la Guerre d’Espagne: «Le Mémorial » attend impatiemment la prise de Bilbao et dénonce la violence anarchiste, l'accaparement du pouvoir par les communistes, générant ainsi une peur viscérale des "rouges". A l'opposé, «Le Cri du Peuple» s'appuie sur les mêmes événements pour lancer un mouvement de ralliement entre communistes et catholiques avec le Comité de Bilbao.

\footnotetext{
NKVD (Narodnyj komissariat vnutrennich del) : police politique de l'URSS.

BENNASSAR, Bartolomé, La Guerre d'Espagne et ses lendemains, cit., p. 185.

AD Loire, PER 317-85 : La Loire républicaine, 8 mai 1937.
} 


\section{Les actions menées dans la Loire}

Enfin, le discours tenu par ces différents journaux ligériens a une fonction de stimulateur. Il provoque et fait perdurer des actions concrètes, il alimente les divisions politiques qui tendent à se cristalliser en réaction à ce discours qui met en exergue une montée des dangers.

\subsection{Des conférences qui divisent les habitants de St-Etienne}

Le 9 avril 1937, devait avoir lieu à Saint-Étienne deux conférences sur la Guerre d’Espagne : le témoignage sur les difficultés endurées par les Républicains de Simone Théry, journaliste et romancière, et une conférence de Philippe Dreux, défendant les franquistes. Cette coïncidence est traité avec ironie par A. Mauperthuis, journaliste à « La Loire Républicaine » : «De nos jours, il y a deux sortes de vérité : la vérité fasciste et la vérité antifasciste. Ce soir, pour les choses espagnoles, il devait y en avoir pour tous les goûts. Les Stéphanois [habitants de St-Etienne] étaient comblés [...]. Eh bien ! Ils resteront tous chez eux, les "fascistes", les "antifascistes" et les autres... Nous n'aurons pas ce soir de conférence sur l'Espagne : ça nous apprendra! »27. En effet, le maire de St-Etienne, Louis Soulié, s'appuyant sur les instructions du gouvernement et du préfet de la Loire, a interdit la conférence de Philippe Dreux. Et, pour éviter un scandale, selon « Le Mémorial », il a reporté à plus tard la conférence de Simone Théry qui devait se tenir à l'Hôtel de ville et avait été organisée par la Maison de la Culture et le Comité féminin contre la guerre et le fascisme ${ }^{28}$ : «Pour montrer par une grimace significative qu'il n'est pas comme on le croit, l'esclave du front communiste, le magistrat de la cité avait - vu les circonstances - remis à une date ultérieure l'exposé de la camarade Simone Théry ${ }^{29}$. Cette analyse de la situation choqua le journaliste de «La Tribune républicaine » : «Notre confrère, trop rapidement informé, parla du soidisant mécontentement de la population stéphanoise. Notre confrère aux prompts réflexes oublia qu'informer est une chose, déformer en est une autre. M. Louis Soulié, maire de St-Etienne, [...] fut retenu par les occupations de sa charge, qui sont

27 AD Loire, PER 317-85 : La Loire républicain, 10 avril 1937.

28 Section locale du Comité mondial des femmes contre la guerre et le fascisme (CMF) qui est présidé par Gabrielle Duchêne et parrainé par le parti communiste français.

29 AD Loire, PER 328-91 : Le Mémorial, 10 avril 1937. 
nombreuses, diverses et impérieuse ».30 Néanmoins, la conférence de Simone Théry eut quand même lieu: "Seulement en bon apôtre du Front rouge, il a permis que la conférence eût lieu à la Bourse du Travail, le même soir » déclare « Le Mémorial » le 10 avril. Apparemment, l'information n'était pas parvenue à A. Mauperthuis, mais elle est confirmée dans le journal du 11 avril. L'article du «Mémorial de conclure » : «La conférencière, dont il ne faut pas nier le talent, parlera des crimes fascistes. Mais elle oubliera les autres, c'est entendu, qui sont pourtant au prologue du terrible drame de la péninsule ». ${ }^{31}$ Et « Le Cri du Peuple » de répondre : « Le catholique Franco bombarde les églises, comme on le voit sur la photo ci-dessus que nous a communiqué Simone Théry. Mais le « Mémorial » se gardera bien de le faire savoir à ses lecteurs».

Ainsi, les conférences sur la Guerre d'Espagne catalysent les opinions politiques qui se radicalisent. L'importation de la guerre civile espagnole dans le département de la Loire par sa médiatisation induit des prises de position binaires : il faut choisir son camp. Toutefois, il est difficile d'évaluer l'emprise de ces débats sur la société ligérienne dans son ensemble : ont-ils passionné surtout des personnes ayant déjà une culture, un engagement politique ou une grande part des ligériens ? Ce questionnement fera l'objet d'autres recherches.

\subsection{L'accueil des civils basques}

Des enfants basques, une centaine, arrivent le 8 mai 1937². Evacués de Bilbao par bateau jusqu'à La Rochelle, ils arrivent à la gare de Châteaucreux de Saint-Étienne où ils sont accueillis par des conseillers municipaux et « un service d'ordre discret assuré par M. Balse, chef de la sûreté et M. Sirven, commissaire du $3^{\text {ème }}$ arrondissement. Les enfants furent conduits au Cercle des cheminots où une collation leur fut offerte, puis ils gagnèrent l'orphelinat du Rez »33. L’information est confirmée par « La Tribune » : une grande part des enfants est par la suite envoyée à la colonie de Montbarnier. Celleci appartient à la municipalité de Saint-Étienne et se trouve en pleine campagne, en Haute-Loire, dans un château vers Yssingeaux. Certains sont placés dans des familles par l’intermédiaire du Comité d’accueil aux réfugiés d'Espagne. Ce-dernier lance

\footnotetext{
30 AD Loire, PER 325-73 : La Loire républicain, 12 avril 1937.

AD Loire, PER 328-91: Le Mémorial, 10 avril 1937.

32 Les archives municipales de Saint-Étienne ne possèdent pas d'archives au sujet de l'accueil des réfugiés espagnols et les archives départementales en ont que sur l'année 1939. Il faudra donc valider ces informations avec celles de Vincennes. Néanmoins, des témoignages édités dans des livres conservés à la Bourse du Travail de Saint-Étienne confirment cet accueil.

33 AD Loire, PER 325-73 : La Loire républicain, 10 mai 1937.
} 
d'ailleurs un appel à la générosité dans « La Tribune Républicaine » du 15 mai : «Les enfants sont, pour la plupart, dans un état physiologique mauvais, sous-alimentés qu'ils sont depuis de longs mois, ils manquent de tout. C'est pourquoi le Comité d'accueil fait plus que jamais appel à la générosité de tous. Envoyez-nous de l'argent, beaucoup d'argent, envoyez-nous des vêtements »34.

Force est de constater l'absence d'article dans «Le Cri du Peuple» au sujet de l'arrivée de réfugiés basques dans le département tant le journal est accaparé par le Comité de Bilbao. Le comité départemental d'accueil aux réfugiés d'Espagne est créé par la CGT, en février 1937. « Ces enfants, avant d'être hébergés chez l'habitant, seront pendant quinze jours à trois semaines, groupés en colonies. A cet effet, la municipalité du Front populaire de ST-Etienne a mis à disposition [...] les colonies scolaires de Pavezin et de Montbarnier. Mieux encore, elle a pris à sa charge l'hébergement de vingt enfants à l'orphelinat du Rez. Le citoyen Soulié, maire, a bien voulu accepter la présidence d'honneur du Comité départemental. »35 Son président, Claudius Buard, est instituteur. Il adhère au PCF en 1924, et est le secrétaire du syndicat unitaire de l'enseignement. Il est chargé de la collecte des dons faits au comité. Des initiatives pour garder des enfants espagnols ont lieu dans les villes des vallées industrielles de l'Ondaine (Firminy) et du Gier (La Ricamarie, Izieux, St-Chamond, Rive-de-Gier), au sud du département. « De nombreux camarades nous demandent, à tout instant, quand arrivera le premier convoi. »36 Mais, alors qu'une centaine d'enfants basques est accueillie, aucun article n'est rédigé à ce sujet.

L'accueil le plus important de réfugiés a lieu un mois plus tard, le 8 juin 1937 : 900 civils basques sont annoncés par « La Tribune Républicaine », le 10 juin. Cette fois-ci des adultes sont présents à côté des enfants. Dans « La Loire Républicaine », l'article est accompagné de 5 photographies montrant l'arrivée de ces réfugiés sur le quai de la gare de Saint-Étienne ainsi qu'une vue de Bilbao ${ }^{37}$. La photographie jointe au discours participe à la construction de l'image du réfugié, avec son baluchon, le regard fermé. « Et bientôt, sur le quai noirâtre de la gare, 400 malheureux réfugiés, traînant leurs hardes, portant de maigres bagages, attendent sans trop d'impatience les ordres des autorités françaises ». Pourtant, la photographie en-dessous, de femmes souriantes, tenant leurs enfants, déconstruit ce cliché. Elle est légendée: «Fières de leur progéniture, ces mamans, oubliant pour une seconde leur misère, arborent un gracieux sourire ».

34 AD Loire, PER 325-73 : La Tribune Républicaine, 15 mai 1937.

5 AD Loire, PER 28 - 6 : Le Cri du Peuple, 11 février 1937.

AD Loire, PER 28 - 6 : Le Cri du Peuple, 18 février 1937.

AD Loire, PER 325-73 : La Loire républicain, 10 juin 1937. 
Fig. 2

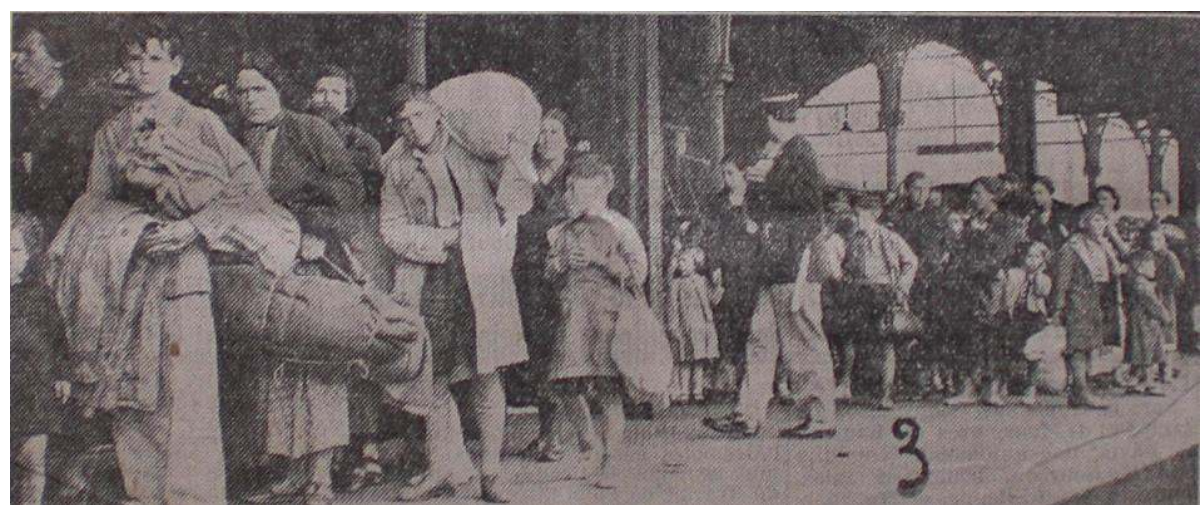

Photographies dans La Loire Républicaine du 10 juin 1937 de l'arrivée de réfugiés basques à St-Etienne

Fig. 3

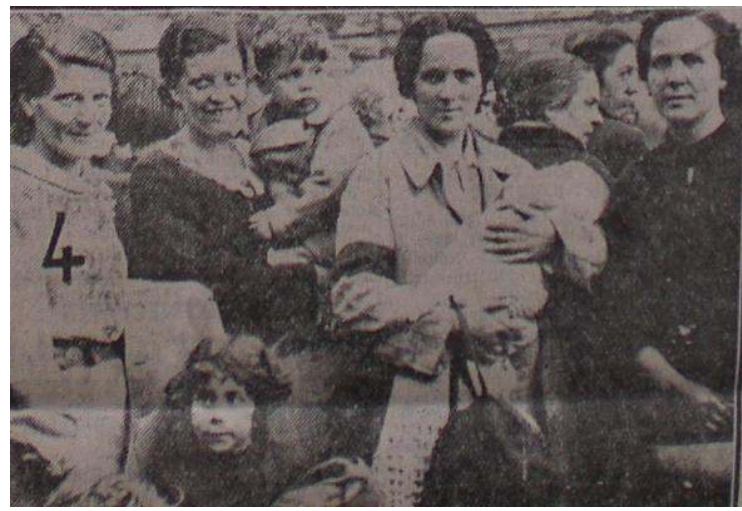

La Loire Républicaine du 10 juin 1937

«La Loire républicaine » fait état de 400 réfugiés à Chantegrillet, dans les bâtiments de l'ancienne école des mines de St-Etienne, ainsi que 580 à Roanne, ville textile du Nord du département. Les archives municipales de Riorges, commune jouxtant Roanne, conservent un dossier attestant de l'accueil de 551 réfugiés basques. Arrivés à 8 h3o à la gare de Roanne, ils ont marché, sous escorte, jusqu'à Riorges où deux usines désaffectées de l'industrie textile allaient servir de lieu d'hébergement. L’une d'elle avait déjà été aménagée (installation de toilettes, d'électricité) lors d'un 
accueil précédant de 121 réfugiés espagnols en septembre 1936. Quant à Saint-Étienne, aucun aménagement n'a été prévu dans les locaux de l'ancienne école des Mines. Le comité d'accueil aux enfants d'Espagne fournit des paillasses et des moïses «pour les enfants de moins de deux ans qui couchaient avec leur mère parterre ${ }^{8}{ }^{8}$. Les comités féminins de Saint-Étienne fournissent du linge, des vêtements, des chaussures.

Cet accueil est source de fierté : « Notre terre est une terre d'asile et de liberté. Ceux qui ont quitté leur patrie pour échapper aux horreurs d'une tragédie infernale, trouveront chez nous l'aide fraternelle ». "Le Mémorial », qui parle uniquement des réfugiés arrivés à St-Etienne, dans un article plus court, au ton plus sec, est moins enthousiaste quant à l'accueil des réfugiés : "La France, pays toujours généreux et hospitalier, reçoit continuellement des réfugiés »39. De plus, « La Loire Républicaine » soulève le problème sanitaire et rassure ses lecteurs : «Toutes précautions ont été prises au débarquement à La Pallice où tout le monde a été examiné et vacciné bien entendu. » De manière insidieuse, ces articles construisent un sentiment de peur : peur de la violence des combats avec l'image prégnante des bombardements de Guernica, peur de la guerre qui pousse les civils à l'exil, panique face à cet afflux de réfugiés démunis, miséreux qui pourraient apporter avec eux des épidémies. «L’Espagne rend la France malade de la peur ${ }^{40}$.

\subsection{Le comité de Bilbao}

Comme il a été constaté précédemment, l'attaque de Bilbao par les nationalistes est utilisé localement par le parti communiste pour créer une union: «[...] les communistes tendent une main fraternelle à tous les ouvriers, paysans, intellectuels, jeunes et vieux, hommes et femmes, décidés à coordonner leurs énergies. Unir, voilà notre mot d'ordre à nous [...]. Unir tous les hommes qui ont soif de justice et de fraternité, et qui rêvent d'instaurer une société meilleure. " ${ }^{41}$ Et cette union réalisée avec la création du Comité de Bilbao, le 15 mai 1937, vise particulièrement les catholiques : «Le peuple basque est catholique. Et nous appelons à collaborer à notre effort tous les catholiques d'ici [...]. Nous n'apportons dans cette action, nous laïques, aucun calcul, aucune prétention, aucun sectarisme. Formons la chaîne de toutes les

38 Témoignage de Claudius Buard, in SANGUEDOLCE, Joseph, Parti pris pour la vie, Lyon, VO Editions, 1993, p. 91.

39 AD Loire, PER 328-91 : Le Mémorial, 9 juin 1937.

40 LABORIE, Pierre, «Les Français des années troubles », Paris, Editions Desclée de Brouwer, 2001, p. 110.

41 AD Loire, PER 28 - 6 : Le Cri du Peuple, article d'Ollier, journal du 28 mai 1937. 
volontés.»42 L'article du 21 mai fait allusion aux tensions qui existent autour de ce projet : «Parce que l'initiative émanait de notre parti, certains s'attendaient à nous voir en réclamer une place prééminente. D’autres redoutaient que nous fassions une "publicité" de ce geste. On a un peu honte, dans de tels moments et pour une telle cause, d'avoir à répondre à de telles questions ». L'appel à l'unité se tourne également vers les socialistes, qui avaient été fort malmenés au début des années 1930 par le PCF. Les événements d'Espagne sont encore une fois exploités localement. L'union des partis communiste et socialiste espagnols, en juin 1937, permet de conclure : « Union d'autant plus nécessaire que l'unité d'action internationale des fascismes, elle, est depuis longtemps réalisée. » 43 Cette politique répond au mot d'ordre de rassemblement défini dans le VII ${ }^{\circ}$ congrès de l'Internationale Communiste en 1935 avec la possibilité de participer à des gouvernements de Front populaire, ce que n'a pas été le cas en France, en mai 1936. Mais, la Guerre d'Espagne semble redonner un élan et cette politique du rassemblement est vivante localement.

Les objectifs du Comité de Bilbao sont clairs, ils sont énoncés dans le journal du 17 mai du «La Loire Républicaine »: «Ce comité rassemblera et acheminera, par les moyens les plus rapides, vers Bilbao, les secours recueillis ». Plusieurs organisations ont rejoint le comité comme "Les femmes contre la guerre", le parti socialiste, les syndicats du textile, des métaux, des mineurs et des amicales de quartiers ouvriers. Le but est de faire partir le plus rapidement au moins trois camions. L'annonce de la création du Comité de Bilbao dans «Le Cri du Peuple» est encadrée par deux photographies : un alignement de cadavres de femmes basques et le chargement d'un navire pour apporter des secours. Elles rendent explicites l'urgence de la situation. Les dons en nature (vivres, linge, vêtements, médicaments) sont à envoyer à Louis Ollier, un des cadres de la section locale du PCF, à la gare de Châteaucreux ou au siège du parti, 45, rue de la République, à Saint-Étienne. Le 28 mai, un article annonce : «Deux camions sont partis! Vite deux autres »44. La collecte des dons se fait désormais dans un local de la cour de l'Hôtel de ville de St-Etienne, des permanences sont tenues de 18h30 à $20 \mathrm{~h}$ du lundi au vendredi et le samedi matin. Le départ de camions est confirmé dans «La Loire Républicaine » et «Le Mémorial », mais est présenté très différemment: «Un convoi de sept camions neufs, chargés d'une marchandise invisible, des vivres nous a-t-on dit, étaient de passage à St-Etienne, hier dans l'aprèsmidi [le 27 mai 1937]. Cinq stationnèrent devant l'hôtel de ville et deux devant l'agence

2 AD Loire, PER 28 - 6 : Le Cri du Peuple, 14 mai 1937.

43 AD Loire, PER 28 - 6 : Le Cri du Peuple, journal du 10 juin 1937.

44 AD Loire, PER 28 - 6 : Le Cri du Peuple, 28 mai 1937. 
Havas. Inutile de dire que son passage chez nous a été abondamment commenté »45. Et effectivement, «Le Mémorial » décrit la même caravane de camions dans son journal du 27 mai. Dans celui du 10 juin, un autre convoi est annoncé : «Hier, à 23 h 30, les promeneurs attardés encore dans la rue Wilson [rue donnant accès à la cour centrale de la Mairie] ont pu voir s'engouffrer, dans la cour de l'Hôtel de Ville, deux superbes camions portant cette inscription "Comité de Bilbao ; offert par le parti communiste". Que viennent faire ces camions dans la maison municipale ? Y recevoir certainement des vivres destinés aux gouvernementaux!» Aucune annonce de ce convoi est faite dans « Le Cri du Peuple». Par contre, du 14 mai au 24 juin, date de l'annonce de la chute de Bilbao, chaque édition du journal fait un bilan exhaustif des dons pour le comité de Bilbao, en énumérant les donateurs et le montant de leurs dons.

Ainsi, la Guerre d'Espagne a-t-elle réellement été exploitée dans la presse ligérienne : elle est matière à créer de l'événement. Sa médiatisation, à l'excès sur certaines périodes, se fait à l'échelle locale, en lien avec les événements, les tensions présentes dans la Loire. Elle alimente des peurs, construit une image de l'exode et des réfugiés dès 1937 , durcit les tensions politiques, et stimule des comportements : de générosité pour certains, de méfiance et de rejet pour d'autres. Une étude comparative avec la presse d'autres départements permettra de compléter cette analyse.

45 AD Loire, PER 325-73 : La Loire républicain, 28mai 1937. 


\section{* L’auteur}

Pascale Moiron est professeur agrégé d'Histoire dans l'enseignement secondaire. Elle est en deuxième année de thèse d'Histoire contemporaine à l'EHESS Paris. Son sujet de recherche porte sur les réfugiés espagnols dans la Loire (1936-1958). Ses travaux sont encadrés par Gérard Noiriel, et rattachés au laboratoire IRIS. De plus, elle est membre de l'association « Adelante » qui rassemble des étudiants dont les axes de recherche se rapportent à la Guerre d'Espagne.

URL: < http://studistorici.com/progett/autori/\#Moiron >

\section{Per citare questo articolo:}

MOIRON, Pascale, «La guerre d'Espagne (sur)exposée dans la presse française. Etude locale de la presse du département de la Loire (42)», Diacronie. Studi di Storia Contemporanea: Spagna Anno Zero: la guerra come soluzione, 29/07/2011,

URL:<http://www.studistorici.com/2011/07/29/moiron_numero_7/ >

\section{Diacronie Studi di Storia Contemporanea $\beta$ www.diacronie.it}

Risorsa digitale indipendente a carattere storiografico. Uscita trimestrale. redazione.diacronie@hotmail.it

Comitato di redazione: Marco Abram - Giampaolo Amodei - Jacopo Bassi - Luca Bufarale - Alessandro Cattunar - Alice De Rensis Barbara Galimberti - Deborah Paci - Fausto Pietrancosta - Martina Sanna - Matteo Tomasoni - Luca Zuccolo

\section{Diritti: gli articoli di Diacronie. Studi di Storia Contemporanea sono pubblicati sotto licenza Creative Commons 2.5} Possono essere riprodotti a patto di non modificarne i contenuti e di non usarli per fini commerciali. La citazione di estratti è comunque sempre autorizzata, nei limiti previsti dalla legge. 